\section{Estudo de base populacional sobre o consumo de medicamentos entre idosos: Projeto Bambuí}

\author{
A population-based study on use \\ of medications by elderly Brazilians: \\ the Bambuí Health and Aging Study (BHAS)
}

\author{
Antônio I. de Loyola Filho 1 \\ Elizabeth Uchoa ${ }^{1}$ \\ Josélia de Oliveira Araújo Firmo ${ }^{1}$ \\ Maria Fernanda Lima-Costa ${ }^{1}$
}

\section{Introdução}

1 Núcleo de Estudos em Saúde Pública e Envelhecimento, Centro de Pesquisas René Rachou, Fundação Oswaldo Cruz/Universidade Federal de Minas Gerais, Belo Horizonte, Brasil.

Correspondência A. I. Loyola Filho Núcleo de Estudos em Saúde Pública e Envelhecimento, Centro de Pesquisas René Rachou, Fundação Oswaldo Cruz/Universidade Federal de Minas Gerais. Av. Augusto de Lima 1715, Belo Horizonte, $M G$ 30190-002, Brasil. aloy@cpqrr.fiocruz.br

\begin{abstract}
This study aimed to assess the prevalence and factors associated with use of prescribed and non-prescribed medications among older adults living in Bambui, Minas Gerais, Brazil. A total of 1,606 (92.2\%) out of 1,742 inhabitants aged $60+$ years were interviewed. Among the participants, 1,281 (79.7\%) and 274 (17.1\%) had used prescribed and non-prescribed drugs in the previous 90 days, respectively. Use of prescribed medications was independently associated with gender (female), age (70-79 and $\geq 80$ ), higher family income, worse health conditions, and physician visits. Non-prescribed medications were negatively associated with physician visits and positively associated with female gender and consultation with a pharmacist. In general, factors associated with the use of prescribed and non-prescribed drugs in this study were similar to those observed in studies conducted in other countries. Meanwhile, our results differ from those of other studies by showing less frequent use of prescribed medications among the poorer elderly. Moreover, our results suggest that selfmedication has been used in place of formal health care in the study community.
\end{abstract}

Elderly; Drug Utilization; Self Medication
O envelhecimento da população vem ocorrendo de forma muito acentuada em países em desenvolvimento, como conseqüência da redução da fecundidade, da mortalidade infantil e do aumento da expectativa de vida nas idades mais velhas. No Brasil, o número de habitantes com sessenta ou mais anos de idade passou de 3 milhões em 1960 para 14 milhões em 2000, devendo atingir 32 milhões em 2025, correspondendo à sexta mais numerosa população idosa no mundo 1 .

Os idosos convivem mais freqüentemente com problemas crônicos de saúde, o que os leva a uma maior utilização de serviços de saúde e a um elevado consumo de medicamentos 2 . Esse consumo elevado de medicamentos acarreta riscos à saúde, sendo diversos os fatores que concorrem para isso. Num aspecto mais geral, destacam-se as modificações na farmacocinética de vários medicamentos em virtude de alterações fisiológicas associadas ao envelhecimento. No campo dos medicamentos prescritos, o aumento de deficits cognitivos e visuais dificulta o reconhecimento do medicamento e um adequado cumprimento da prescrição terapêutica por parte do idoso. São observadas ainda prescrições sem suporte científico apropriado. Tais fatores, muitas vezes na presença de doenças concomitantes e do consumo simultâneo de um maior número de fár- 
macos, aumentam a probabilidade de ocorrência de reações adversas e interações medicamentosas 2,3.

Existem, ainda, os problemas derivados do consumo de medicamentos sem prescrição (automedicação), uma prática cada vez mais freqüente entre populações, independentemente dos diferentes contextos sócio-econômicos e culturais em que elas estejam inseridas 4 . $\mathrm{O}$ crescimento da automedicação tem sido favorecido pela multiplicidade de produtos farmacêuticos lançados no mercado e pela publicidade que os cerca, pela simbolização da saúde que o medicamento pode representar e pelo incentivo ao autocuidado, além de outros fatores 4,5. Assim, a automedicação acrescenta aos riscos relacionados ao consumo de medicamentos prescritos a possibilidade de se mascarar ou retardar o diagnóstico de condições mais sérias, dificultando a atuação do médico, pois nem sempre o paciente menciona essa prática durante a consulta médica 5,6. Desse modo, impõe-se um duplo ônus aos serviços de saúde: além dos gastos com a atenção farmacêutica, superiores àqueles decorrentes de consultas médicas, novas despesas originam-se do atendimento a enfermidades relacionadas ao uso inadequado de fármacos 2 .

Estudos epidemiológicos de base populacional têm mostrado que, entre idosos, existem diferenças no padrão de consumo de medicamentos prescritos e não prescritos, sendo aqueles mais consumidos do que estes 7,8,9,10,11,12. Os medicamentos que atuam sobre o sistema cardiovascular são a categoria terapêutica mais utilizada entre os prescritos, ao passo que, entre os não prescritos, prevalecem os analgési$\cos 7,8,13$. Em geral, o consumo de medicamentos prescritos é associado ao sexo feminino 12,14, às faixas etárias mais elevadas 8, à utilização de serviços de saúde (visitas médicas e hospitalização) 8,14 e a um pior estado de saúde 8,10,12. Por sua vez, o consumo de medicamentos não prescritos é observado com maior freqüência no sexo feminino, sendo esta a variável mais consistentemente associada a tal prática 15.

Nos países em desenvolvimento, estudos caracterizando o consumo de medicamentos entre idosos são raros. No Brasil, Miralles \& Kimberlin 16 realizaram esse estudo entre idosos residentes em três bairros da cidade do Rio de Janeiro, distintos quanto à condição sócioeconômica. A utilização de medicamentos prescritos foi mais de duas vezes superior ao consumo de medicamentos não prescritos $(78,0 \%$ versus $34,0 \%$ ). Nesse estudo, verificou-se que sexo (feminino), idade, qualidade do atendimento médico recebido (aceitável/satisfató- ria), percepção da saúde (pior) e um maior número de sintomas percebidos estiveram independentemente associados ao uso de medicamentos prescritos. A automedicação, por sua vez, esteve associada a um menor número de sintomas percebidos, a uma menor capacidade para a compra dos medicamentos e a uma maior disposição em aceitar aconselhamento leigo sobre medicamentos 16 .

No presente trabalho, serão utilizados dados da linha de base da coorte de Bambuí, Minas Gerais, Brasil 17, para descrever a prevalência do consumo de medicamentos prescritos e não prescritos entre idosos residentes na comunidade. Um objetivo adicional do estudo é examinar as características sócio-demográficas, indicadores da condição de saúde e do uso de serviços de saúde associados ao uso de medicamentos prescritos e não prescritos nessa população.

\section{Metodologia}

\section{Área estudada}

O Município de Bambuí contava, em 1996, com 21.187 habitantes $(70,0 \%$ vivendo na cidade de Bambuí). A esperança de vida ao nascer na localidade era 70,2 anos, a taxa de mortalidade infantil era 48,4/1.000 nascidos vivos e o índice de desenvolvimento humano era igual 0,70 . As principais causas de morte entre os habitantes no município eram acidente vascular cerebral, doença de Chagas e doença isquêmica do coração (taxas de mortalidade $=118,0 ; 61,4$ e 42,5 por $100 \mathrm{mil}$ habitantes, respectivamente). Maiores detalhes podem ser vistos em Lima-Costa et al. 17 .

\section{População estudada}

A população de estudo foi identificada por meio de um censo conduzido pela equipe do projeto entre novembro e dezembro de 1996 17. Todos os residentes na cidade com sessenta ou mais anos em 1o de janeiro de 1997 foram selecionados para participar da linha de base $(n=1.742)$.

\section{Coleta de dados}

As informações para este trabalho foram obtidas utilizando o questionário BHAS (Bambuí Health and Aging Study). As entrevistas foram realizadas no domicílio do participante entre os meses de janeiro e agosto de 1997 e tiveram uma duração média de noventa minutos. Diante da impossibilidade de algum entrevistado responder ao questionário, em virtude de defi- 
cit cognitivo ou de algum problema de saúde, recorreu-se a um respondente próximo, o qual não respondeu a perguntas que requeressem julgamento pessoal, como a percepção da saúde. Para maiores detalhes ver Lima-Costa et al. 17.

\section{Variáveis do estudo}

A variável dependente deste estudo é uso de medicamentos. As seguintes perguntas foram utilizadas para a obtenção de informações sobre uso de medicamentos: (1) "Nos últimos três meses, você tomou algum remédio? [Não importa se foi receitado por médico ou não nem a razão pela qual está tomando o remédio]"; (2) “Qual o(s) nome (s) do(s) remédio(s) que está tomando ou tomou nos últimos três meses?"; (3) "Este(s) remédio(s) foi (foram) prescrito(s) por: médico/outro". Solicitou-se, ainda, ao entrevistado a apresentação da receita e/ou embalagem do remédio consumido. Os medicamentos foram classificados de acordo com o sistema de codificação anatômico-terapêutico-químico do World Health Organization Collaborating Centre for Drugs Statistics Methodology 18.

Foram considerados três conjuntos de variáveis exploratórias: sócio-demográficas (sexo, idade, estado conjugal, número de pessoas residentes no domicílio, número de anos completos de escolaridade, renda familiar mensal em salários mínimos da época), indicadores da condição de saúde (percepção da própria saúde, história de diagnóstico médico para algumas doenças/condições selecionadas, interrupção das atividades rotineiras por problemas de saúde nas duas últimas semanas, ter estado acamado nas duas últimas semanas) e indicadores da utilização de serviços de saúde (nos últimos 12 meses, número de visitas a um médico, consulta a um farmacêutico/atendente e internações hospitalares). Além dessas, foi considerada a filiação a plano privado de saúde.

\section{Análise dos dados}

Com base nas perguntas sobre uso de medicamentos, foram constituídos quatro grupos de comparação: (1) consumo exclusivo de medicamentos prescritos; (2) consumo exclusivo de medicamentos não prescritos; (3) consumo simultâneo de medicamentos prescritos e não prescritos e (4) nenhum consumo de medicamento (grupo referência). A análise dos dados foi desenvolvida em duas etapas: inicialmente foi realizada uma análise bivariada, utilizando-se o teste do qui-quadrado de Pearson; a seguir, foi realizada a análise multivariada, utilizando-se o método de regressão logística mul- tinomial 19. Nesta análise, os grupos de consumidores de medicamentos (as três categorias da variável evento) são simultaneamente comparados ao grupo de referência. As variáveis que apresentaram associação com a variável dependente em nível $<$ 0,20 na análise bivariada foram incluídas no modelo logístico inicial. Permaneceram no modelo final aquelas variáveis associadas à variável dependente em nível $<0,05$. A análise dos dados foi realizada utilizando-se o programa Stata versão 7.0.

\section{Resultados}

Dos 1.742 idosos residentes na cidade de Bambuí, 1.606 participaram do presente estudo. Destes, 1.383 relataram ter consumido pelo menos um medicamento nos últimos três meses: $1.109(69,1 \%)$ haviam consumido exclusivamente medicamentos prescritos; 102 (6,4\%), medicamentos não prescritos, e 172 (10,7\%) haviam consumido simultaneamente medicamentos prescritos e não prescritos.

Os medicamentos com ação sobre o aparelho cardiovascular (código C) foram os mais consumidos $(36,2 \%)$ entre os prescritos, principalmente os diuréticos (código C03) e cardioterápicos (código C01). Seguiram-se os medicamentos que atuam sobre o sistema nervoso (código N) (25,5\%), sobretudo analgésicos (código N02) e psicolépticos (código N05). Entre os não prescritos, os medicamentos que atuam sobre o sistema nervoso central (código N) foram os mais consumidos $(37,1 \%)$, na quase totalidade analgésicos (código N02), seguidos de medicamentos do trato alimentar e metabolismo (código A) (21,3\%), especialmente vitaminas (código A11) e antiácidos (código A02), e de medicamentos do sistema músculo-esquelético (código M) (12,0\%).

Na Tabela 1 está apresentada a distribuição do consumo de medicamentos prescritos e não prescritos, segundo variáveis sócio-demográficas. Sexo, idade, estado conjugal e renda familiar mensal apresentaram associações significantes $(\mathrm{p}<0,05)$ com uso de medicamentos na análise bivariada.

O uso de medicamentos esteve significativamente associado $(\mathrm{p}<0,05)$ a todos os indicadores da condição de saúde investigados na análise bivariada, quais sejam, percepção da saúde, número de doenças ou condições crônicas, interrupção de atividades de rotina por problemas de saúde nas duas últimas semanas e ter estado acamado neste período (Tabela 2).

Número de consultas médicas, ocorrência de internação hospitalar e consulta ao farma- 
Consumo de medicamentos prescritos e não prescritos segundo algumas variáveis sócio-demográficas selecionadas.

Bambuí, Minas Gerais, Brasil, 1997.

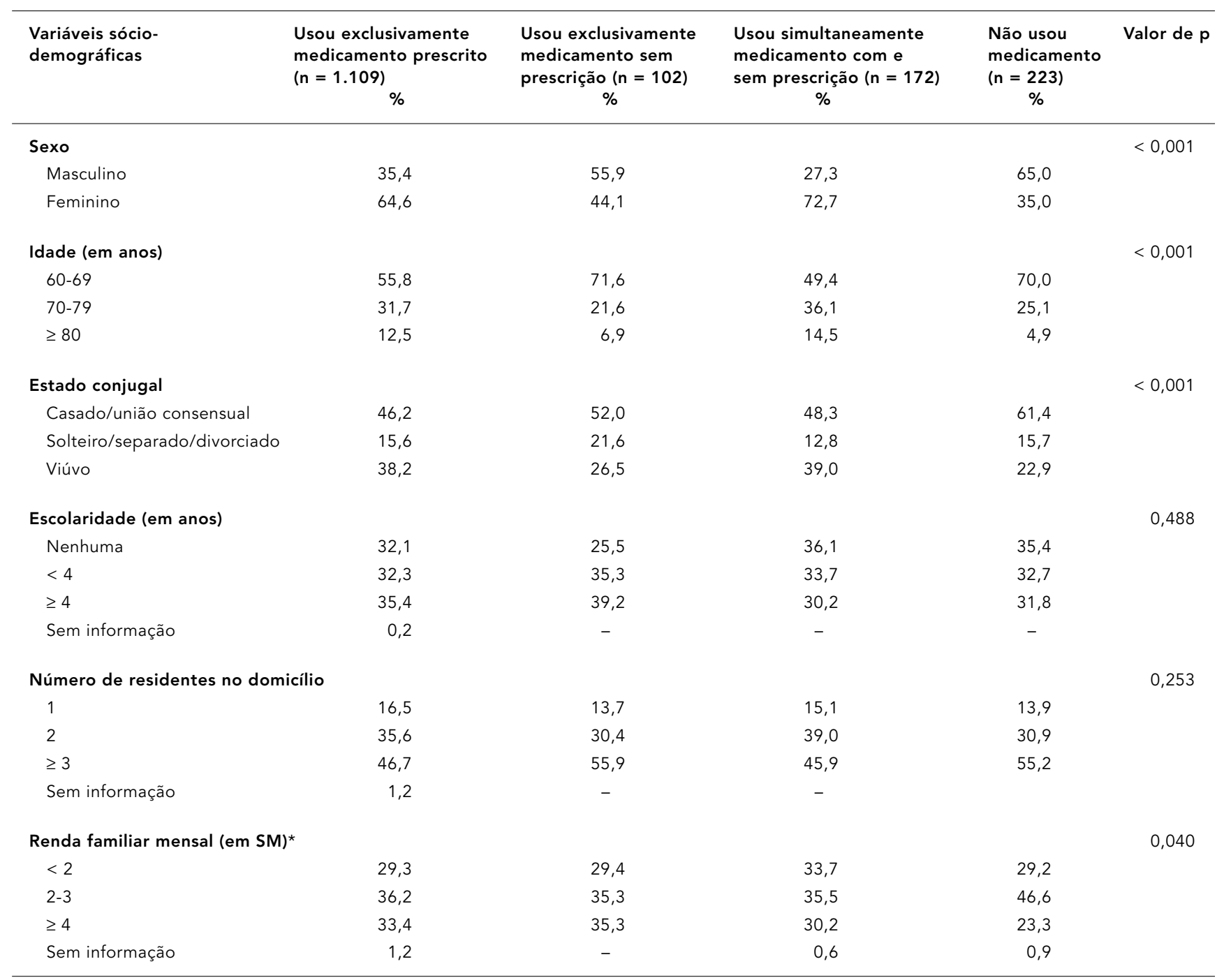

* $\mathrm{SM}=$ salários mínimos nacionais vigentes à época (1SM = US\$120,00); valor de p: qui-quadrado de Pearson, desconsiderado "sem informação" para o cálculo.

cêutico/atendente, nos últimos 12 meses, além de filiação a plano privado de saúde apresentaram associações significantes $(\mathrm{p}<0,05)$ com uso de medicamentos na análise bivariada (Tabela 2).

Os resultados finais da análise multinomial dos fatores associados ao uso de medicamentos prescritos e não prescritos estão apresentados na Tabela 3. Associações positivas com o uso de medicamentos prescritos foram encontradas com sexo (feminino), idade (70-79 e $\geq 80$ anos), renda familiar mensal ( $\geq 4$ salários mínimos), percepção da própria saúde (razoável, ruim/muito ruim), número de doenças ou condições crônicas (1 e $\geq 2$ ), interrupção das ativi- dades de rotina por problemas de saúde nas duas últimas semanas e número de consultas médicas nos últimos 12 meses (1, 2-3 e $\geq 4)$.

Associações independentes e positivas com o uso de medicamentos não prescritos foram encontradas para o sexo feminino e consulta a um farmacêutico/atendente nos últimos 12 meses; associação independente e negativa foi encontrada para número de consultas médicas nos últimos 12 meses.

O grupo de usuários simultâneos de medicamentos prescritos e não prescritos apresentou resultados bastante semelhantes ao verificado para o grupo de usuários de medicamen- 
Consumo de medicamentos prescritos e não prescritos, segundo algumas variáveis indicadoras do estado de saúde, utilização de serviços de saúde e filiação a plano de saúde privado. Bambuí, Minas Gerais, Brasil, 1997.

\begin{tabular}{|c|c|c|c|c|c|}
\hline $\begin{array}{l}\text { Variáveis sócio- } \\
\text { demográficas }\end{array}$ & $\begin{array}{l}\text { Usou exclusivamente } \\
\text { medicamento prescrito } \\
(n=1.109) \\
\%\end{array}$ & $\begin{array}{l}\text { Usou exclusivamente } \\
\text { medicamento sem } \\
\text { prescrição }(n=102) \\
\qquad \%\end{array}$ & $\begin{array}{l}\text { Usou simultaneamente } \\
\text { medicamento com e } \\
\text { sem prescrição ( } n=172) \\
\%\end{array}$ & $\begin{array}{l}\text { Não usou } \\
\text { medicamento } \\
(n=223) \\
\%\end{array}$ & Valor de $p$ \\
\hline \multicolumn{2}{|l|}{ Percepção da própria saúde } & & & & $<0,001$ \\
\hline Muito boa/boa & 18,6 & 40,2 & 14,5 & 46,2 & \\
\hline Razoável & 46,4 & 47,1 & 53,5 & 40,8 & \\
\hline Ruim/muito ruim & 28,1 & 9,8 & 29,1 & 10,3 & \\
\hline Desconhecida* & 6,9 & 2,9 & 2,9 & 2,7 & \\
\hline \multicolumn{2}{|l|}{$\begin{array}{l}\text { Número de condições/ } \\
\text { doenças crônicas }\end{array}$} & & & & $<0,001$ \\
\hline Nenhum & 18,5 & 56,9 & 14,0 & 5,2 & \\
\hline 1 & 35,4 & 36,3 & 38,4 & 30,5 & \\
\hline$\geq 2$ & 46,0 & 6,9 & 47,7 & 14,4 & \\
\hline Sem informação & 0,1 & - & - & - & \\
\hline \multicolumn{2}{|c|}{$\begin{array}{l}\text { Interrupção de atividades } \\
\text { de rotina por problemas de saúde } \\
\text { nas duas últimas semanas }\end{array}$} & & & & $<0,001$ \\
\hline Não & 80,3 & 92,2 & 77,9 & 95,5 & \\
\hline Sim & 19,7 & 7,8 & 21,5 & 4,5 & \\
\hline Sem informação & 0,1 & - & 0,6 & - & \\
\hline \multicolumn{2}{|l|}{$\begin{array}{l}\text { Ter estado acamado nas duas } \\
\text { últimas semanas }\end{array}$} & & & & $<0,001$ \\
\hline Não & 87,5 & 97,1 & 89,0 & 97,8 & \\
\hline Sim & 12,4 & 2,9 & 10,5 & 2,2 & \\
\hline Sem informação & 0,2 & - & 0,6 & - & \\
\hline \multicolumn{2}{|l|}{$\begin{array}{l}\text { Número de consultas médicas } \\
\text { nos últimos } 12 \text { meses }\end{array}$} & & & & $<0,001$ \\
\hline Nenhuma & 10,6 & 59,8 & 14,5 & 48,4 & \\
\hline 1 & 19,3 & 22,6 & 17,4 & 30,0 & \\
\hline $2-3$ & 31,1 & 11,8 & 36,1 & 14,4 & \\
\hline$\geq 4$ & 39,0 & 5,9 & 32,0 & 7,2 & \\
\hline \multicolumn{2}{|c|}{$\begin{array}{l}\text { Consulta ao farmacêutico/atendente } \\
\text { nos últimos } 12 \text { meses }\end{array}$} & & & & $<0,001$ \\
\hline Não & 95,0 & 92,2 & 83,7 & 97,3 & \\
\hline Sim & 5,0 & 7,8 & 16,3 & 2,7 & \\
\hline \multicolumn{2}{|l|}{$\begin{array}{l}\text { Internação hospitalar nos } \\
\text { últimos } 12 \text { meses }\end{array}$} & & & & $<0,001$ \\
\hline Não & 73,7 & 96,1 & 73,8 & 90,6 & \\
\hline Sim & 26,3 & 3,9 & 26,2 & 9,4 & \\
\hline \multicolumn{2}{|l|}{ Filiação a plano privado de saúde } & & & & 0,037 \\
\hline Não & 79,1 & 88,2 & 80,8 & 85,2 & \\
\hline Sim & 20,9 & 11,8 & 19,2 & 14,8 & \\
\hline
\end{tabular}

* Outra pessoa respondeu à entrevista; ** Hipertensão arterial, doença coronariana, doença de Chagas, diabetes e reumatismo; valor de $\mathrm{p}=$ qui-quadrado de Pearson, desconsiderado "sem informação" para o cálculo. 
Tabela 3

Resultados finais da análise multivariada dos fatores associados ao uso de medicamentos prescritos e não prescritos.

Bambuí, Minas Gerais, Brasil, 1997.

\begin{tabular}{|c|c|c|c|c|c|c|}
\hline \multirow[t]{2}{*}{ Variáveis } & \multicolumn{2}{|c|}{$\begin{array}{l}\text { Uso exclusivo de } \\
\text { medicamentos prescritos }\end{array}$} & \multicolumn{2}{|c|}{$\begin{array}{l}\text { Uso exclusivo de } \\
\text { medicamentos sem prescrição }\end{array}$} & \multicolumn{2}{|c|}{$\begin{array}{l}\text { Uso simultâneo de } \\
\text { medicamentos prescritos } \\
\text { e sem prescrição }\end{array}$} \\
\hline & OR & IC95\% & OR & IC95\% & OR & IC95\% \\
\hline \multicolumn{7}{|l|}{ Sexo } \\
\hline Masculino & 1,00 & & 1,00 & & 1,00 & \\
\hline Feminino & 2,40 & $1,69-3,41$ & 1,70 & $1,03-2,79$ & 3,82 & $2,36-6,19$ \\
\hline \multicolumn{7}{|l|}{ Idade (em anos) } \\
\hline $60-69$ & 1,00 & & 1,00 & & 1,00 & \\
\hline $70-79$ & 1,61 & $1,10-2,37$ & 0,84 & $0,47-1,50$ & 2,32 & $1,42-3,82$ \\
\hline$\geq 80$ & 3,43 & $1,67-7,04$ & 1,63 & $0,60-4,48$ & 5,80 & $2,50-13,43$ \\
\hline \multicolumn{7}{|c|}{ Renda familiar mensal em SM* } \\
\hline$<2$ & 1,00 & & 1,00 & & 1,00 & \\
\hline $2-3$ & 1,03 & $0,69-1,55$ & 0,77 & $0,43-1,39$ & 1,01 & $0,59-1,73$ \\
\hline$\geq 4$ & 2,77 & $1,74-4,42$ & 1,78 & $0,94-3,36$ & 2,56 & $1,41-4,66$ \\
\hline \multicolumn{7}{|l|}{ Percepção da saúde } \\
\hline Muito boa/boa & 1,00 & & 1,00 & & 1,00 & \\
\hline Razoável & 1,69 & $1,15-2,49$ & 1,66 & $0,98-2,82$ & 2,59 & $1,15-4,64$ \\
\hline Ruim/muito ruim & 2,00 & $1,13-3,56$ & 1,47 & $0,60-3,58$ & 2,85 & $1,33-6,09$ \\
\hline Desconhecida** & 4,32 & $1,66-11,24$ & 1,37 & $0,32-5,93$ & 2,42 & $0,63-9,33$ \\
\hline \multicolumn{7}{|c|}{ 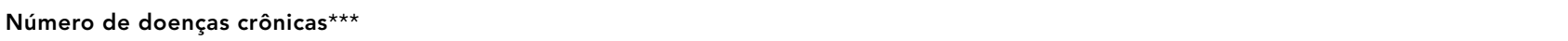 } \\
\hline Nenhum & 1,00 & & 1,00 & & 1,00 & \\
\hline 1 & 2,78 & $1,89-4,11$ & 1,16 & $0,69-1,98$ & 3,58 & $1,97-6,51$ \\
\hline$\geq 2$ & 4,60 & $2,85-7,44$ & 0,43 & $0,17-1,08$ & 5,73 & $2,95-11,13$ \\
\hline \multicolumn{7}{|c|}{$\begin{array}{l}\text { Interrupção das atividades de rotina } \\
\text { por problemas de saúde nas duas } \\
\text { últimas semanas }\end{array}$} \\
\hline Não & 1,00 & & 1,00 & & 1,00 & \\
\hline Sim & 2,26 & $1,11-4,63$ & 2,01 & $0,74-5,48$ & 2,63 & $1,17-5,89$ \\
\hline \multicolumn{7}{|c|}{$\begin{array}{l}\text { Número de consultas médicas } \\
\text { nos últimos } 12 \text { meses }\end{array}$} \\
\hline Nenhuma & 1,00 & & 1,00 & & 1,00 & \\
\hline 1 & 1,94 & $1,28-2,96$ & 0,53 & $0,30-0,96$ & 1,05 & $0,54-2,03$ \\
\hline $2-3$ & 5,24 & $3,21-8,54$ & 0,53 & $0,25-1,14$ & 3,10 & $1,58-6,06$ \\
\hline$\geq 4$ & 9,36 & $5,07-17,29$ & 0,52 & $0,18-1,48$ & 3,77 & $1,72-8,25$ \\
\hline \multicolumn{7}{|c|}{$\begin{array}{l}\text { Consulta ao farmacêutico/atendente } \\
\text { nos últimos } 12 \text { meses }\end{array}$} \\
\hline Não & 1,00 & & 1,00 & & 1,00 & \\
\hline Sim & 1,54 & $0,60-3,94$ & 4,27 & $1,40-13,02$ & 7,01 & $2,57-19,08$ \\
\hline
\end{tabular}

* $\mathrm{SM}=$ salários mínimos nacionais vigentes à época (1SM = US\$120,00); ** Outra pessoa respondeu à entrevista;

$\star \star \star$ Hipertensão arterial, doença coronariana, doença de Chagas, diabetes e reumatismo; OR = odds ratio,

IC95\% = intervalo de confiança ao nível de 95\%: ajustados pelo método de regressão logística multinomial por todas

as variáveis listadas na tabela. Para essa análise, o grupo de referência foi o de não-usuários de medicamento;

1.589 respondentes foram incluídos na análise. 
tos prescritos. O uso concomitante de medicamentos com e sem prescrição médica esteve independente e positivamente associado a: sexo feminino, idade (70-79 e $\geq 80$ ), renda familiar mensal ( $\geq 4$ salários mínimos), pior percepção de saúde (razoável, ruim/muito ruim), número de doenças crônicas ( 1 e $\geq 2$ ), impossibilidade de realizar atividades de rotina nos últimos 15 dias por causa de problemas de saúde (sim), número de consultas médicas $(2-3 \mathrm{e} \geq 4)$ e consulta ao farmacêutico/atendente (sim) nos últimos 12 meses.

\section{Discussão}

O consumo dos medicamentos prescritos verificado neste trabalho foi superior ao que se encontrou em idosos residentes em países desenvolvidos $8,10,12$ e semelhante ao observado na cidade do Rio de Janeiro 16. Por outro lado, o uso de medicamentos não prescritos entre idosos bambuienses foi menor que o observado nos estudos acima mencionados. Os principais medicamentos prescritos (ação sobre o aparelho cardiovascular) e não prescritos (analgésicos) foram os mesmos observados em países desenvolvidos $7,8,13,20,21,22$.

Nossos resultados confirmam a observação de que as mulheres idosas aparecem, em distintas populações, como maiores consumidoras de medicamentos prescritos 7,10,12,13,14,15,22. O maior consumo de medicamentos prescritos entre as mulheres pode estar relacionado à maior presença de condições crônicas e queixas entre elas e à maior utilização de serviços de saúde 10,21,23, fatores que aumentam a probabilidade da prescrição médica. No presente trabalho, a associação entre sexo feminino e consumo de medicamento prescrito persistiu mesmo após ajustamentos por consultas ao médico e estado de saúde. Laukkanen et al. 24 lembram que as mulheres são as principais responsáveis pelos cuidados com a saúde na família e, por isso, estão mais familiarizadas com os medicamentos. Isso talvez contribua para uma maior propensão à automedicação entre elas, especialmente na abordagem de problemas de saúde já vivenciados.

O efeito da idade sobre o consumo de medicamentos entre idosos é diferenciado para os medicamentos com e sem prescrição médica. Estudos epidemiológicos longitudinais 9,11 e transversais 7,10,13,16 evidenciaram um significativo aumento do uso de medicamentos prescritos com o avanço da idade. Esse quadro pode ser causado pelo aumento das doenças com a idade, pelo aumento de visitas a um ou mais médicos e pela necessidade de utilização de combinações medicamentosas 21 . Por outro lado, a influência da idade no uso de medicamentos não prescritos é controversa. Diversos trabalhos mostraram que a automedicação em idosos não é afetada pela idade 7,13,20,22, ao passo que, em outros, foi observado aumento 9 ou diminuição 11,14 da automedicação com o aumento da idade. No presente trabalho, foi observado o aumento no consumo de medicamentos prescritos com a idade, mas a prevalência da automedicação permaneceu estável ao longo das faixas etárias.

Em países desenvolvidos, a renda ou classe social não parece influenciar o consumo de medicamentos prescritos entre idosos 12,14,21, mas na cidade do Rio de Janeiro verificou-se um menor consumo desses medicamentos em bairros mais pobres 16 . Nossos resultados estão de acordo com essas últimas observações. Em Bambuí, o consumo de medicamentos prescritos entre idosos apresentou associação independente com a renda mensal domiciliar, sendo significativamente mais alto entre aqueles com renda mais elevada. Esse resultado reforça observações realizadas em um estudo qualitativo entre idosas bambuienses, mostrando a importância da situação sócio-econômica para a aquisição e manutenção da dose prescrita do medicamento 25.

Como era de se esperar, neste trabalho os indicadores da condição de saúde apresentaram as associações mais fortes com uso de medicamentos prescritos, as quais foram graduadas para número de doenças/condições crônicas. Esses achados são consistentes com observações realizadas em outras populações $8,9,10$, $12,14,16,23$.

Com relação ao uso de serviços de saúde, chama atenção a forte associação entre maior número de consultas médicas e uso de medicamentos prescritos, assim como a prática menos freqüente da automedicação entre aqueles que realizaram consultas médicas. Estudos realizados em países desenvolvidos mostraram que a consulta médica está fortemente associada ao consumo de medicamentos prescritos $8,11,14,21$, pois aumenta a probabilidade de gerar uma prescrição medicamentosa. No Brasil, uma maior aceitação dos serviços médicos apresentou-se positivamente relacionada ao consumo de medicamentos prescritos entre idosos residentes no Rio de Janeiro 16. Em relação ao consumo de medicamentos sem prescrição, alguns pesquisadores 26,27 consideram as associações negativas entre automedicação e consulta médica um indicativo da substituição da atenção formal à saúde pela automedi- 
cação. A possibilidade de que a automedicação constitua, em Bambuí, um mecanismo de substituição da atenção formal à saúde é reforçada pela associação independente existente entre esta e consulta a um farmacêutico/atendente. É necessário ressaltar que no Brasil, tanto em pequenas comunidades, como em cidades maiores, a farmácia desempenha um importante papel na rede informal de atenção à saúde $16,28,29,30$.

$\mathrm{Na}$ presente pesquisa, os procedimentos clássicos para evitar a presença de vieses em inquéritos epidemiológicos foram adotados. A identificação de participantes foi censitária e desenvolveram-se esforços a fim de obter uma ampla participação da população no estudo, garantindo sua validade interna 17 . O período utilizado para a obtenção de informações sobre o uso de medicamentos foi de noventa dias, o que, em comparação a outros estudos que utilizaram períodos menos longos, pode ter levado a uma superestimação do consumo de medicamentos (em virtude do uso acumulado de medicamentos no período) ou a uma subestimação desse consumo (por causa de pro-

\section{Resumo}

Investigou-se a prevalência e fatores associados ao consumo de medicamentos prescritos e não prescritos entre idosos (60 anos ou mais). Participaram deste trabalho 1.606 (92,2\%) dos 1.742 idosos residentes na cidade de Bambuí, Minas Gerais, Brasil. Entre os participantes, 1.281 (79,7\%) e 274 (17,1\%) haviam consumido medicamentos prescritos e não prescritos nos últimos noventa dias, respectivamente. O consumo de medicamentos prescritos esteve associado ao sexo feminino, idade (70-79 $e \geq 80$ anos), renda familiar (maior), estado de saúde (pior) e número de consultas médicas (maior). O uso de medicamentos não prescritos apresentou associação negativa com consulta médica e associação positiva com sexo (feminino) e consulta a um farmacêutico. Os fatores associados ao consumo de medicamentos prescritos e não prescritos verificados neste trabalho foram semelhantes aos observados em estudos conduzidos em outros países. Diferentemente deles, nosso estudo mostrou um menor consumo de medicamentos prescritos entre idosos com pior situação sócio-econômica. Nossos resultados sugerem, ainda, que a automedicação entre idosos esteja sendo utilizada em substituição à atenção formal à saúde.

Idosos; Uso de Medicamentos; Automedicação blemas de memória). Entretanto, acreditamos que a adoção de procedimentos padronizados para a coleta de dados (com referência ao treinamento dos entrevistadores e à formulação das perguntas), além da solicitação de apresentação da embalagem e/ou receita para o medicamento consumido, tenha contornado eventuais problemas de memória.

Em resumo, os resultados deste trabalho mostram que, de uma maneira geral, os tipos de medicamentos prescritos e não prescritos para a população idosa estudada são muito semelhantes aos observados em países desenvolvidos, assim como os fatores associados a essas práticas. Todavia, dois resultados chamam atenção: (1) a associação entre a pior situação sócio-econômica e o menor uso de medicamentos prescritos e (2) os indícios sugestivos de que a automedicação possa estar substituindo a atenção formal à saúde entre idosos. Novos estudos são necessários para permitir uma compreensão mais profunda dos mecanismos envolvidos nessas associações e suas conseqüências para as condições de saúde da população idosa.

\section{Colaboradores}

A. I. Loyola Filho foi o responsável pela análise dos dados e redação do artigo. J. O. A. Firmo organizou e coordenou todo o trabalho de campo e participou da discussão dos resultados. E. Uchoa participou da discussão dos resultados e fez uma revisão crítica final do texto. M. F. Lima-Costa orientou o trabalho, supervisionou a análise e a redação e fez a revisão crítica final do texto. 


\section{Referências}

1. Lima-Costa MF, Veras R. Saúde pública e envelhecimento [Editorial]. Cad Saúde Pública 2003; 19: 700-1.

2. Tamblyn R. Medication use in seniors: challenges and solutions. Therapie 1996; 51:269-82.

3. Rozenfeld S. Prevalência, fatores associados e mau uso de medicamentos entre os idosos: uma revisão. Cad Saúde Pública 2003; 19:717-24.

4. Loyola Filho AI, Uchoa E. Automedicação: motivações e características de sua prática. Rev Méd Minas Gerais 2002; 12:219-27.

5. Lowe NK, Ryan-Wenger NM. Over-the-counter medications and self-care. Nurse Pract 1999; 24: 34-44.

6. Barnett NL, Denham MJ, Francis SA. Over-thecounter medicines and the elderly. J R Coll Physicians Lond 2000; 34:445-6.

7. Helling DK, Lemke JH, Semla TP, Wallace RB, Lipson DP, Cornoni-Huntley J. Medication use characteristics in the elderly: the Iowa 65+ Rural Health Study. J Am Geriatr Soc 1987; 35:4-12.

8. Stoller EP. Prescribed and over-the-counter medicine use by the ambulatory elderly. Med Care 1988; 26:1149-57.

9. Jylhä M. Ten-year change in the use of medical drugs among the elderly - a longitudinal study and cohort comparison. J Clin Epidemiol 1994; 47:69-79.

10. Woo J, Ho SC, Yuen YK, Lau J. Drug use in an elderly Chinese population: prevalence and associated factors. Gerontology 1995; 41:98-108.

11. Fillenbaum GG, Horner RD, Hanlon JT, Landerman LR, Dawson DV, Cohen HJ. Factors predicting change in prescription and nonprescription drug use in a community-residing black and white elderly population. J Clin Epidemiol 1996; 49:58793.

12. Espino DV, Lichtenstein MJ, Hazud H, Fabrizio DBA, Wood RC, Goodwin J, et al. Correlates of prescription and over-the-counter medication usage among older Mexican Americans: the Hispanic EPESE study. Established Population for the Epidemiologic Study of the Elderly. J Am Geriatr Soc 1998; 46:1228-34.

13. Chrischilles EA, Foley DJ, Wallace RB, Lemke JH, Semla TP, Hanlon JT, et al. Use of medications by persons 65 and over: data from the Established Populations for Epidemiologic Studies of Elderly. J Gerontol 1992; 47:M137-44.

14. Fillenbaum GG, Hanlon JT, Corder EH, ZiqubuPage T, Wall Jr. WE, Brock D. Prescription and nonprescription drug use among black and white community-residing elderly. Am J Public Health 1993; 83:1577-82.

15. Hanlon JT, Fillenbaum GG, Ruby CM, Gray S, Bohannon A. Epidemiology of over-the-counter drug use in community dwelling elderly: United States perspective. Drugs Aging 2001; 18:123-31.
16. Miralles MA, Kimberlin CL. Perceived access to care and medication use among ambulatory elderly in Rio de Janeiro, Brazil. Soc Sci Med 1998; 46:345-55.

17. Lima-Costa MFF, Uchoa E, Guerra HL, Firmo JOA, Vidigal PG, Barreto SM. The Bambuí Health and Aging Study (BHAS): methodological approach and preliminary results of a population-based cohort study of the elderly in Brazil. Rev Saúde Pública 2000, 34:126-35.

18. WHO Collaborating Centre for Drug Statistics Methodology. Anatomical therapeutic chemical (ATC) classification index with defined daily doses (DDDs). Oslo: World Health Organization; 2000.

19. Hamilton LC. Interpreting multinomial logistic regression. Stata Technical Bulletin 1993; 13:24-8.

20. May FE, Stewart RB, Hale WE, Marks RG. Prescribed and nonprescribed drug use in an ambulatory elderly population. South Med J 1982; 75:522-8.

21. Lassila HC, Stoehr GP, Ganguli M, Seaberg EC, Gilby JE, Belle SH, et al. Use of prescription medications in an elderly rural population: the MoVIES Project. Ann Pharmacother 1996; 30:589-95.

22. Stoehr GP, Ganguli M, Seaberg EC, Echement DA, Belle S. Over-the-counter medication use in an older rural community: the MoVIES Project. J Am Geriatr Soc 1997; 45:158-65.

23. Hershman DL, Simonoff PA, Frishman WH, Preston F, Aronson MK. Drug utilization in the old old and how it relates to self-perceived health and all-cause mortality: results from the Bronx Aging Study. J Am Geriatr Soc 1995; 43:356-60.

24. Laukkanen P, Heikkinen E, Kauppinen M, Kallinen $M$. Use of drugs by non-institutionalized urbans finns born in 1904-1923 and the association of drug use with mood and self-rated health. Age Ageing 1992; 21:343-52.

25. Uchoa E. Contribuições da antropologia para uma abordagem das questões relativas à saúde do idoso. Cad Saúde Pública 2003; 19:849-53.

26. Bush PJ, Osterweis M. Pathways to medicine use. J Health Soc Behav 1978; 19:179-89.

27. Conn V. Older adults: factors that predict the use of over-the-counter medication. J Adv Nurs 1991; 16:1190-6.

28. Haak H. Padrões de consumo de medicamentos em dois povoados da Bahia (Brasil). Rev Saúde Pública 1989; 23:143-51.

29. Queiroz MS. Estratégias de consumo em saúde entre famílias trabalhadoras. Cad Saúde Pública 1993; 9:272-82.

30. Rozemberg B. O consumo de calmantes e o "problema de nervos” entre lavradores. Rev Saúde Pública 1994; 28:300-8.

Recebido em 22/Mar/2004

Aprovado em 04/Nov/2004 